\title{
The Review of General Wireless Communication Signal to S - band Pulsed Radar Fuze Signal Interference
}

\author{
Wang Pengfei \\ Xi'an Research Institute of High Technology, Xi'an710025, China \\ e-mail:396954319@qq.com
}

\begin{abstract}
Based on the frequency characteristics of pulsed radar fuze signal and general wireless communication signal, the paper tries to analyze the interference that the latter on the former. By analyzing the working principle, working characteristics and interference environment of the pulsed radar fuze system and the frequency characteristics, carrier bandwidth, transmit power and modulation mode of several typical general wireless communication systems, it further points out that the possibility of interference' existence. And at the same time, it gives out the method steps and verification methods of interference decision.
\end{abstract}

Key words: wireless communication; radar fuze; interference; summary

\section{Introduction}

The pulsed radar fuze is used in China's nuclear missile detonation system which transmission and reception frequencies are basically set on the S-band of the radio frequency. In the past, since the S-band was a military band, the radar fuze would not be interfered by other wireless devices especially the interference by the public communication equipment. But with the rapid development of wireless communication technology, there have been more and more common wireless communication signal frequency into the S-band range including the 3G/4G signals of the mobile phone, the wireless network communication signals and digital cordless telephone signals, whose spectrum has covered the S-band, and the transmission power is high, which may cause the fuze system to exploding ahead or misfiring. Therefore, it is of great military significance and application value to study the interference problem of general wireless communication signal to S-band pulsed radar fuze. The article mainly analyzes the interference of 3G and 4G signals to pulsed radar fuze.

\section{Introduction to Pulsed Radar Fuze}

\subsection{Working Principle of Pulsed Radar Fuze}

Pulsed radar fuze is a kind of radio fuze that transmits the high frequency pulse signal with a certain repetition period, which can provide non-fuzzy height information. The working principle is shown in Figure $1^{[1]}$. The pulse-modulated high-frequency oscillating waves are transmitted to the space via the antenna, and the echo signal is generated after running into the target. Depending on the delay time of the target echo pulse relative to the transmitted pulse, the distance from the target to the warhead can be derived. The distance diagram is shown in Figure 2.

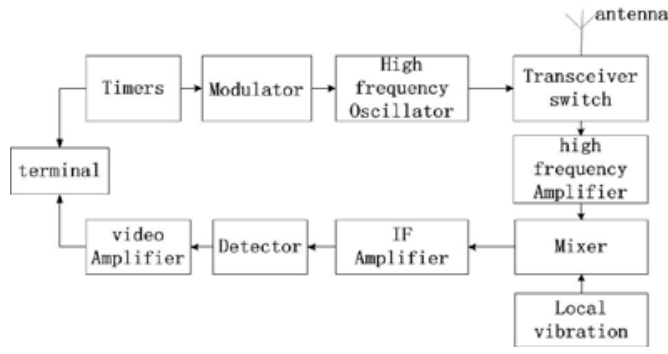

Figure 1. Block diagram of pulsed radar fuze

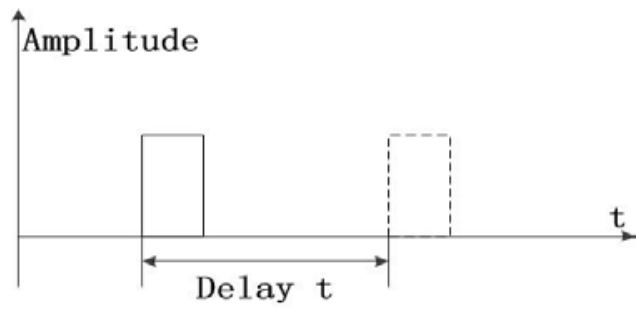

Figure 2. Pulse Ranging Schematic 


\subsection{Pulsed Radar Fuze Working Characteristics}

Because of the volume requirements of nuclear weapons, the pulsed radar fuze system applied to it must be miniaturized. In order to meet this requirement, the fuze antenna is designed to be shared. That is, when the signal is transmitted, the signal can not be received at the same time. It is bound to have a blind spot within the time $t_{c}$ of the transmitted pulse, and the presence of the blind spot is not allowed for the radar fuze. If the transceiver antenna is used separately, the limited distance between the transceiver antenna, will inevitably lead to a considerable leakage of the transmission signal. The gate must be taken to avoid the fuze signal's interference to the echo signal, which will not only complicate the fuze system, but will also lead to tactical limitations, and blind spots at close range. In order to solve this problem, the direct detection of pulse radar fuze should be out of the potential, but at the same time it provides the conditions for the external radio interference of the same frequency, adjacent frequency and intermodulation .

\subsection{Interference Environment of Pulsed Radar Fuze}

It can be seen from the principle and operating characteristics of the pulse radar fuze, the pulse radar fuze is interfered internally and externally. The internal interference is the inherent noise of the radar fuze, which mainly includes the thermal noise of the components, the mechanical vibration noise and so on. The external interference can be divided into two categories: natural disturbance and artificial disturbance. Natural disturbances include cosmic electrical interference, industrial electromagnetic interference, the frequency interference of electronic equipment, interference by the cloud rain and lightning interference and so on. Artificial disturbances mainly include passive interference such as false target, metal dipole and active jamming including throwing disturbance, answering interference, aiming interference, blocking interference and swept interference. It can be seen that the universal wireless communication signal may become a kind of natural disturbance factor and interfere the pulse radar fuze.

\subsection{The Current Situation of The Pulsed Radar Fuze Anti - jamming Technology}

Fuze anti-jamming technology is mainly to use the expansion or manufacture of the difference between the interfering signal and the beneficial echo signal to achieve the purpose of separating them. These differences include signal energy, spatial characteristics, temporal characteristics, operating frequency domain, polarization direction and signal characteristics, etc. At present, radar fuze anti-jamming technology mainly aims to improving the receiver signal to interference ratio (such as increasing transmit power, reducing receiver bandwidth, increasing transmit antenna gain, reducing transmission system losses, using linearly polarized receiving antennas, etc.) , increasing the transmit signal concealment (such as selecting a special operating frequency, selecting the style of pulse amplitude modulation and phase modulation, choosing remote access), enhancing the self-protection of transmission channel (such as transceiver antenna azimuth selection, distance door selection and receiver interference cancellation processing), increasing signal processing gain(such as correlation detection, coherent accumulation, and move target detection), etc. However, these anti-jamming techniques are used only for internal noise interference from pulsed radar fuze and for man-made interference such as repressive and deceptive. Up to now, there has been no study of whether the general-purpose wireless communication signal will interfere the pulse radar fuze.

\section{Interference Analysis of General Wireless Communication Signals on S - band Pulsed Radar Fuze}

\subsection{Interference Style Analysis}

3.1.1 The interference of single - mode signal to S - band pulsed radar fuze

a) Interference of WCDMA signal to $\mathrm{s}$ - band pulsed radar fuze

WCDMA (Wideband Code Division Multiple Access) is a 3G communication technology originated in Europe and Japan. It is adopted by China Unicom in China. Its communication frequency band is set at $1940 \mathrm{MHz}-1955 \mathrm{MHz}$ (uplink), $2130 \mathrm{MHz}-2145 \mathrm{MHz}$ (downlink) ${ }^{[2]}$. Its 
carrier bandwidth is $5 \mathrm{MHz}$, the base station transmit power is up to $33 \mathrm{dBm}$, the transmit antenna gain is generally $18 \mathrm{dBi}$. The communication model is shown in Figure 3.

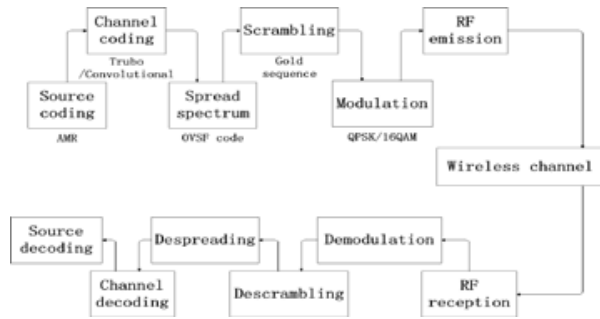

Figure 3. WCDMA communication model block diagram

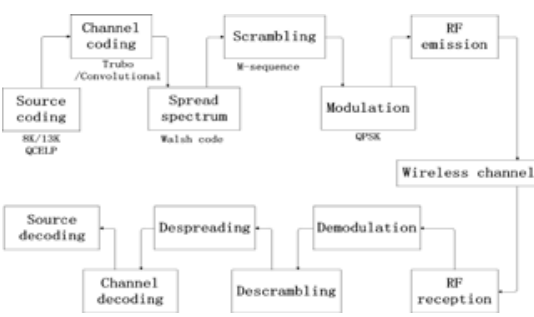

Figure 4. CDMA2000 communication model block diagram

The data containing the information is encoded by AMR to obtain the corresponding data bit stream, and then the symbol stream is obtained after being encoded and intertwined by the convolutional code or Trubo code. After the symbols stream through the OVSF code, the signal band is widened and the rate reaches the corresponding chip rate. The use of Gold serial code for scrambling code can enhance the ability of signal anti-multiple access interference, and then converted to analog signals through the QPSK or 16QAM modulation, which is launched after been amplified by the RF unit.

From the characteristics of WCDMA system and communication principle, it can be seen that the RF signal emitted from the base station antenna occupies the wide frequency band of the S-band and has high power, so it is likely to cover all or part of the pulse radar fuze on the S-band Echo signal. It becomes a stronger noise signal entering the passband of the fuze receiver, which affects the receiver's detection of the echo signal.

b) Interference of CDMA2000 signal to $s$ - band pulse radar fuze

CDMA2000 is a 3G mobile communication standard proposed by Qualcomm North America, which is adopted by China Telecom in China. Its spectral range is $1920 \sim 1935 \mathrm{MHz}$ (uplink) / $2110 \sim$ $2125 \mathrm{MHz}$ (downlink) ${ }^{[3]}$, the base station transmission power is generally $43 \mathrm{dBm}$, and the maximum antenna gain is $18 \mathrm{dBi}$. The communication model is shown in Figure 4.

The data containing the information is first encoded by the voice encoder to obtain the corresponding data bit stream, and then it is encoded by Turbo code or convolutional code to obtain the corresponding symbol stream, which will avoid the appearance of error code. The bandwidth of the signal after spreading with Walsh code is increased to 2 to 43 times the original, the rate reaches the chip rate. The chip is scrambled with M-sequence codes to improve the ability of signal anti-multiple access interference. Finally, the QPSK-modulated chip is converted to an analog signal which is transmitted by radio frequency modulation.

From the analysis of transmission power, spectrum characteristics and communication principle of the CDMA2000 system, it can be concluded that the signal transmitted from the RF transmitting unit in the downlink also occupies the wide frequency band of the S-band and has higher power. Therefore, it is likely to become a strong interference signal at the radar fuze receiver to affect the fuze receiver's detection of its own echo signal.

c) Interference of TD-SCDMA signal to s-band pulsed radar fuze

TD-SCDMA (Time Division Synchronous Code Division Multiple Access) is proposed by China's third-generation mobile communication standard, used by the China Mobile. Its spectrum range is $2010 \mathrm{MHz}-2025 \mathrm{MHz}$, the carrier bandwidth is $1.6 \mathrm{MHz}$, the base station's transmit power is generally $43 \mathrm{dBm}$, the maximum transmission power of the mobile terminal is $30 \mathrm{dBm}^{[4]}$. The communication model is shown in Figure 5. 


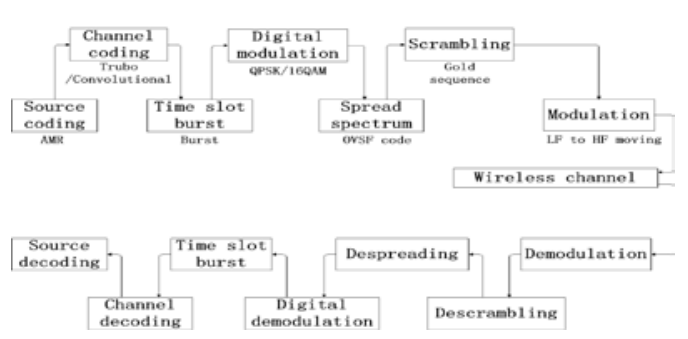

Figure 5. TD-SCDMA communication model block diagram

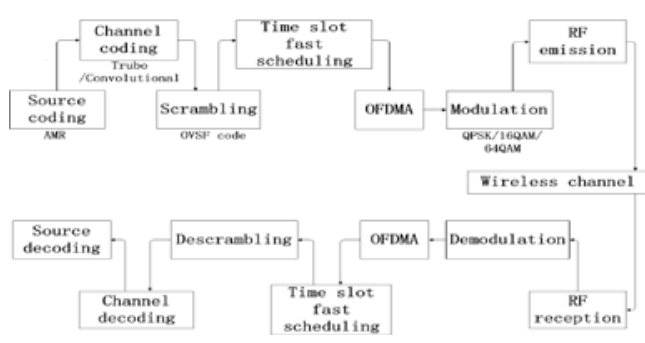

Figure 6. TD-LTE communication model block diagram

The data containing the information is encoded by AMR to generate the corresponding data bit stream, and then the convolutional code or Trubo code is used to improve the reliability of communication. The data bit stream is input to time slot burst unit for synchronization, and then modulated by QPSK or 16QAM to generate the corresponding symbol stream. The corresponding symbol stream is amplified by the OVSF code and the rate is equal to the rate of the chip, and then use the Gold sequence code to scrambling the chip to enhance the ability of anti-multiple access interference, and finally send out the signal through the RF transmitter unit.

From the power characteristics and spectrum range of the TD-SCDMA system, it can be seen that the signals emitted from the RF transmitting unit occupy the wide band of the S-band, both in the uplink and downlink, and have higher power. So the S-band pulsed radar fuze echo signal is likely to be completely covered by these signals, and affect the detection of the fuze receiving system.

\section{d) Interference of TD-LTE signal to pulsed radar fuze}

TD-LTE (Long Time Evolution) is the fourth-generation mobile communication technology and standard jointly developed by Alcatel-Lucent, Nokia Siemens Networks, Datang Telecom, Huawei Technologies, ZTE and China Mobile ${ }^{[5]}$. It uses a time-division duplex technology, that is, the uplink and downlink signals can share the same band. Its base station transmit power is up to $46 \mathrm{dBm}$, and the mobile terminal transmit power is up to $30 \mathrm{dBm}$. The communication model is shown in Figure 6 .

From the TD-LTE spectrum range, the TD-LTE system characteristics and the communication principle, it can be concluded that the spectrum of the signal transmitted from the radio frequency transmitting unit in its downlink and uplink not only occupies the wide band of the S-band, but also has higher power. It is likely to cause the S-band radar fuze signal to be covered in all or part of the mode signal coverage so that the fuze receiver can not be detected.

\subsubsection{Interference of Multi - mode synthesized signal to pulse radar fuze}

It can be seen from the frequency band used in each mode signal, WCDMA and CDMA2000 downlink signal band are adjacent, and both use FDD mode. Therefore, when two relatively close base stations transmit signals simultaneously and the guard band between them is not wide enough, it is easy to interfere with each other to generate new signals. If the frequency of the signal is the same as or similar to that of the S-band pulse radar fuze, it is likely to become the noise signal, which is difficult to be distinguished, enters the frequency range of the fuze receiver and affects the receiver to detect the echo signal.

\subsection{Interference Judging Step}

In order to determine the anti-jamming capability of a radar fuze, it is common to use the criteria such as efficiency, power, information, line improvement factor ${ }^{[6]}$. And to study the interference problem of the general wireless communication signal to the S-band pulse radar fuze it is advised to take the power criterion based on the analysis and comparison of their frequencies. That is, by comparing the wireless communication signal at the fuze receiver and the pulsed radar fuze signal power to determine whether the interference exists. 


\subsubsection{The establishment of the general wireless signal propagation model}

a) Single - mode signal propagation model

In the transmission process, the radio signal, suffers from attenuation due to spatial diffusion, and the phenomena such as reflection, refraction, scattering, diffraction and absorption in spatial propagation would cause other losses. Single-mode signal propagation diagram as shown in Figure 7. Received signal power received can be expressed as:

$$
P_{r}=\frac{\left(P_{s} G_{s}-P_{L}\right)}{4 \pi R^{2}} A_{r} F_{r}^{2} \gamma_{p} \frac{\Delta f_{1}}{\Delta f_{2}}
$$

In the formula (1) where $P_{s}$ is the signal transmit power, $G_{s}$ is the transmit antenna gain, $P_{L}$ is the path loss power, $R$ is the distance between the transmitter and the receiver, $A_{r}$ is the effective receiving area of the receiving antenna, $\gamma_{p}$ is the polarization mismatch coefficient of the transmit and receive antennas, $\Delta f_{1}$ is the bandwidth of the receive antenna, $\Delta f_{2}$ is the signal bandwidth of the transmitting antenna, $F_{r}$ is the directivity function of the receiving antenna.

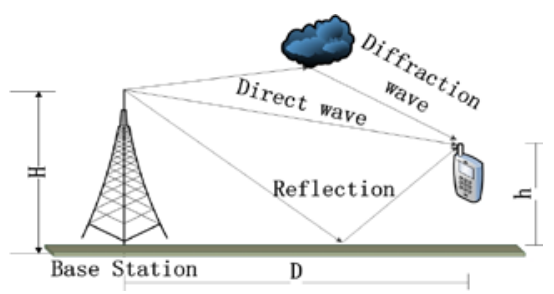

Figure 7. Single-mode signal propagation diagram

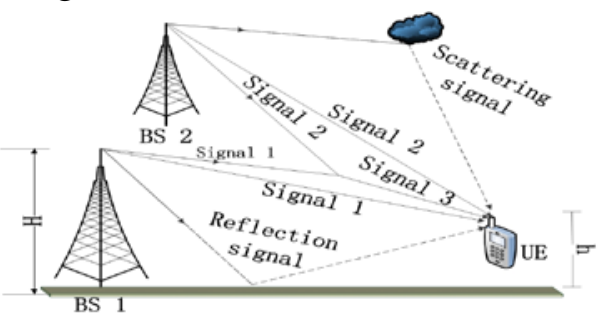

Figure 8. Multi-mode signal propagation diagram

b) Multimode signal propagation model

When the receiving system is covered by two or more different mode base stations, the signal arriving at the receiver will produce a number of harmonic combinations of frequency components due to the non-linearity of the receiving system. The combined frequency components close to the frequency of the desired signal in the middle of them will pass through the receiver, creating intermodulation interference to the useful signal. The multi-mode signal propagation is shown in Figure 8.

It is assumed that the signal expression of the combined frequency component which is close to the frequency of the useful signal received by the receiver is:

$$
S(t)=A \cos (\omega t+\varphi)
$$

Its power expression is:

$$
P_{r}^{\prime}=\frac{1}{T} \int_{-T / 2}^{T / 2} s^{2}(t) d t
$$

\subsubsection{The model of pulsed radar fuze received echo signal}

From the principle of pulsed radar fuze available, the received echo signal power $P_{A s}$ at the pulse radar fuze receiver can be expressed as:

$$
P_{A s}=\frac{\frac{P_{t} D_{t} F_{t}^{2}-P_{R}}{4 \pi R^{2}} A_{0}-P_{R}}{4 \pi R^{2}} A_{r} F_{r}^{2}
$$

In the formula (4), $P_{t}$ is the radiated power of the fuze transmitting antenna, $D_{t}$ is the directional coefficient of the transmitting antenna, $F_{t}$ is the directional function of the transmitting antenna, $F_{r}$ is the directional function of the fuze receiving antenna, $A_{0}$ is the effective area of ground reflection, $A_{r}$ is the effective receiving area of the fuze receiving antenna, $R$ is the distance between fuze and target; $P_{R}$ is the loss of electromagnetic wave in the atmosphere.

\subsubsection{Interference determination}


On the basis of the analysis frequency, it is possible to determine whether the interference exists by determining the power ratio of the wireless communication signal and the pulse radar fuze signal received at the fuze receiver. ${ }^{[6]}$ The ratio can be expressed as:

$$
\delta=\frac{P_{A s}}{P_{r}+P_{s}} \text { or } \delta^{\prime}=\frac{P_{A s}}{P_{r}^{\prime}+P_{s}}
$$

In the formula (5), $P_{s}$ is the total noise power of the fuze system and can be expressed by the equivalent noise temperature $T_{e}{ }^{[6]}$. And there are

$$
T_{e}=\left(F_{n}-1\right) T_{0}
$$

In the formula (6), $F_{n}$ is the noise figure. In general, its value range is $0 \mathrm{~dB}<F_{n}<10 \mathrm{~dB}$, its relation with the receiver's sensitivity can be expressed as:

$$
P_{\min }=4 K T_{0} B_{n} F_{n} \Omega \frac{C}{N}
$$

In the formula (7), $P_{\min }$ is the sensitivity of the receiver; $\mathrm{K}$ is the Boltzmann constant; $T_{0}$ is the thermodynamic temperature at room temperature, $T_{0}=290 \mathrm{~K} ; B_{n}$ is the equivalent noise bandwidth, and its value is the signal bandwidth; $\Omega$ is the receiver input resistance; $\frac{C}{N}$ is the limiter input-side threshold carrier-to-noise ratio. (The typical value is $12 \mathrm{~dB}$.)

There is

$$
F_{n}=\frac{P_{\min }}{4 K T_{0} B_{n} \Omega \frac{C}{N}}
$$

The formula (5) can be transformed into:

$$
\delta=\frac{P_{A s}}{\mathrm{P}_{\mathrm{r}}+\left(\frac{P_{\min }}{4 K T_{0} B_{n} \Omega \frac{C}{N}}-1\right) T_{0}}
$$

or

$$
\delta^{\prime}=\frac{P_{A s}}{\mathrm{P}_{r}^{\prime}+\left(\frac{P_{\min }}{4 K T_{0} B_{n} \Omega \frac{C}{N}}-1\right) T_{0}}
$$

That is, when the missile enters the wireless communication signal energy coverage, and $\delta$ or $\delta^{\prime} \geq 1$, the pulse radar fuze is basically not disturbed by the wireless communication signal; and when $\delta$ or $\delta^{\prime} \leq 1$, the wireless communication signal may cause interference to the pulsating radar fuze.

\subsubsection{Interference verification}

a) Modeling of Universal Wireless Communication System

According to the model block diagram of WCDMA, CDMA2000, TD-SCDMA and TD-LTE system, as shown in Figures 3, 4, 5 and 6, the simulation model of Simulink is established by Simulink function module in Matlab simulation environment. The signal waveforms of WCDMA, CDMA2000 downlink signals, TD-SCDMA, TD-LTE uplink and downlink signals are simulated and added to the input port of the pulse radar fuze receiver as external noise respectively.

b) Modeling of pulsed radar fuze system

According to the schematic diagram of pulse radar fuze system, as shown in Fig. 1, Simulink simulation model is established by Simulink function module in Matlab simulation environment, as shown in Fig.9. To set the initial conditions of the pulse radar fuze can obtain the waveform of the echo signal of the pulse radar fuze when there is or no universal wireless communication signal. By comparing the difference between the two waveforms, you can find that if the receiver can not detect the echo signal, you can determine the existence of interference; otherwise the interference does not exist. 


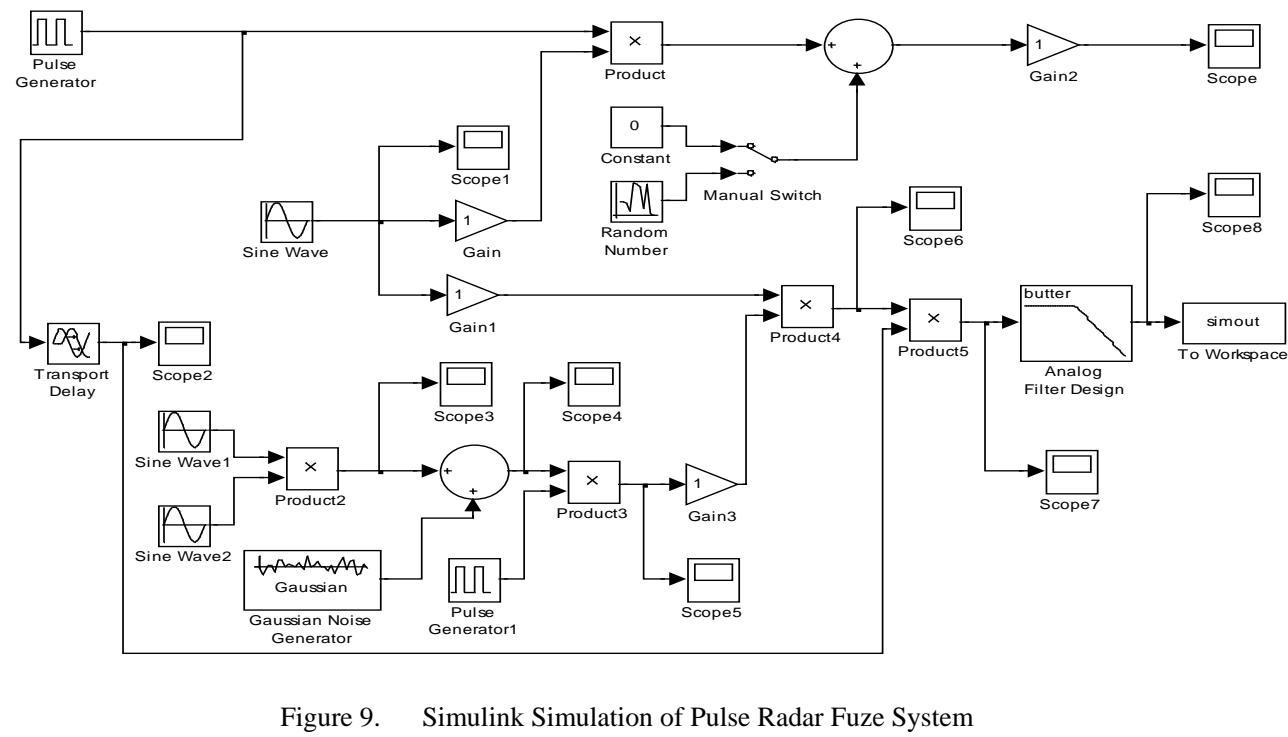

\section{CONCLUSION}

Based on the characteristics of universal wireless communication signals and S - band pulsed radar fuze, it points out that the former may cause interference to the latter, which has important military significance and application value. The possibility of the existence of the interference is further determined by analyzing the working principle, working characteristic, the interference environment of the pulsed radar fuze system and the waveform parameters, modulation mode of several typical general wireless communication signal. At the same time, it finds that the power criterion can be used to judge the existence of interference and the method of computer Matlab modeling and simulation can be used to verify the judgment result, which provides the methods and steps for the further research on the concrete problem.

\section{REFERENCES}

[1] Cui Zhanzhong, Song Shihe. Principle of Proximity Fuze (Third Edition) [M]. Beijing: Beijing Institute of Technology Press, 2009.

[2] LI Ze, etc. Study on anti-active noise jamming performance of pulsed Doppler fuze [J] .Technology, 2015,36 (6): 1002.

[3] Research on Coexistence of WCDMA and CDMA2000 Interference [D]. Beijing: Beijing University of Posts and Telecommunications, 2007.

[4] Ji Changpeng, Li Xu.TD-SCDMA and WiMAX Integrated Downlink Interference Analysis [J]. World Science and Technology Research and Development, 2010,32 (3): 285-286.

[5] LIU Li, CAO Gen, ZHANG Xin. Test Interference Coexistence Analysis of TD-LTE System with WCDMA and TD-SCDMA System [J] .Guangdong Communications Technology, 2010,30 (7): 16-24.

[6] Zhang Qingtai. Radio fuze general design principle [M]. National Defense Industry Press, 1985. 